\title{
SARS Another Emerging Disease
}

\author{
Charles John Palenik
}

Severe acute respiratory syndrome, SARS, is an illness that has been recently reported in Asia, North America, Europe and Africa. SARS appears to be a new disease. The first known case of atypical pneumonia occurred on 16 November 2002 in Foshan City, Guangdong Province, China. However, its significance was not known until later. The disease soon spread to the rest of the Guangdong Province and then further points in South East Asia. The World Health Organization (WHO) started to track the disease actively during mid-February 2003.

From 1st November 2002 to 3rd July 2003, a total of 8439 probable SARS cases were reported to the WHO from 29 countries, including four cases in the United Kingdom and 73 in the United States. There have been 812 deaths (case-fatality proportion $=9.62 \%$ ) reported, with no SARS-related fatalities occurring in the United Kingdom or the United States.

The WHO recently reported the world population should be SARS-free by the end of July 2003. However, the WHO warns the disease could emerge from animal reservoirs in China again next winter. The common belief is that SARS jumped from animals to humans in southern China late last year.

Information on SARS changes daily. Readers are directed to Table 1 for a listing of valuable websites for the latest reports. It is difficult to obtain important and timely information on any emerging diseae today without access to the Internet.

\section{ILLNESS}

A set of criteria has been developed for SARS (Table II). Incubation typically lasts two to seven days. However, ten day incubations have also been noted. Illness usually starts with a prodrome of fever. Temperatures above $38^{\circ} \mathrm{C}\left(100.4^{\circ} \mathrm{F}\right)$ can be present. Fever is often associated with chills, headaches, malaise and myalgia. At the onset of illness some persons have mild respiratory symptoms. Rashes and neurologic or gastrointestinal findings are usually absent.

After three to seven days, the lower respiratory phase begins with the onset of a dry, non-productive cough or dyspnoea. Hypoxemia can then develop. In 10-20\% of cases, illness is severe enough to require intubation and mechanical ventilation. Severity of illness can vary greatly from mild through to fatal.

No specific treatment recommendations can be made at this time. Therapy should include coverage for organisms associated with any community-acquired pneumonia of unclear aetiology, including agents with activity against both typical and atypical respiratory pathogens. Treatment choices may be influenced by severity of the illness. Infectious disease consultation is recommended.

\section{VIROLOGY}

It is currently agreed that a novel coronavirus is the major causative agent of SARS. Coronaviruses are single-stranded RNA viruses that have lipid envelopes with club-shaped projections and include some known to cause respiratory symptoms in humans. The SARS virus, however, is unlike any other known coronavirus. Multiple laboratories have consistently isolated the virus from SARS patients from several

This is the author's manuscript of the article published in final edited form as:

Palenik, C. J. (2003). SARS Another Emerging Disease. Dental update, 30(6), 290-292.

https://doi.org/10.12968/denu.2003.30.6.290 
countries. Reactivity with antibodies has been noted in specimens from SARS patients, but could not be detected in hundreds of uninfected persons.

Other pathogens could serve as cofactors in the SARS disease process. Human metapneumovirus (hMPV) has also been found in respiratory specimens and antibodies against hMPV are present in serum of some SARS patients. Also, there is evidence of dual infection with hMPV and the new coronavirus. The significance of hMPV in SARS remains unclear.

\section{VIRAL SPREAD}

The primary way that SARS appears to spread is by close person-to-person contact. Most cases of SARS have involved people who cared for or lived with someone with SARS, or had direct contact with infectious material (for example, respiratory secretions) from a person who had SARS. Potential ways in which SARS can be spread include touching the skin of other people or objects that are contaminated with infectious droplets and then touching eyes, nose, or mouth. This can happen when someone who is sick with SARS coughs or sneezes droplets onto themselves, other people, or nearby surfaces. Droplets are relatively large and can usually travel only a short distance (a meter or less) before they settle. It also is possible that SARS can be spread more broadly through the air or by other ways that are currently not known.

It has been suggested that 'hypercontagious' persons have been involved with the transmission of SARS. One such person has been implicated in the spread of infection to more than 100 persons. Most new cases have been reported among healthcare workers ( $\mathrm{HCW}$ ) and their families. About $25 \%$ of the 800 SARS cases reported in Hong Kong involved occupationally acquired infections of healthcare workers. Five deaths occurred.

\section{INFECTION CONTROL}

HCWs treating suspected cases of SARS should use standard precautions, including hand hygiene together with airborne (e.g. N-95 respirators) and contact (e.g. gowns and gloves) precautions. Until the mode of disease transmission is better known, eye protection should also be worn. HCWs possibly exposed to SARS should be vigilant for fever or respiratory symptoms. If they develop, immediate medical evaluation should be sought. Exposed HCWs that develop symptoms should stay at home and not go to work. Of course, exposure does not always mean infection.

Dentists should not treat known or suspected cases of SARS. Instead, the suspect patient should be sent directly to a healthcare facility or a physician for diagnosis and care. The case should immediately be reported to the local health department. Until more is known about the transmission of the disease, SARS patients should remain at home for seven days after discharge from the hospital. Only then should they seek dental treatment.

\section{SARS, ANOTHER EMERGING DISEASE}

SARS is the first severe and readily transmissible new disease to emerge in the twenty-first century. Although much remains poorly understood, SARS has demonstrated a marked ability to spread within densely populated areas and via international travel routes.

One physician, infected while treating patients in his hometown in southern China, brought the virus to a hotel in Hong Kong. Although he only spent one night, guests and visitors to the ninth floor became 
infected and proceeded to seed hospital outbreaks throughout South East Asia. Hotel guests also spread the disease along international air travel systems to even more distance destinations. The result was the formation of 'hot zones' of infection within Hong Kong, Vietnam, Singapore and Toronto. Unsuspecting hospital personnel started to treat ill patients without complete barrier protection. Initial outbreaks were then followed by a series of secondary transmission outside of the healthcare facilities.

SARS poses a serious threat for several reasons. There is no preventive vaccine, nor is there effective treatment. HCWs were forced to use seemingly ancient methodologies - isolation and quarantine. Also, coronaviruses are notorious for frequent mutations, thus calling into question the nature of future outbreaks and the chances of rapid vaccine development. Initial symptoms are non-pathognomonic and rather common, thus hindering early diagnosis and patient sequestering. A significant number of SARS cases require intensive hospital care. This places significant strain on healthcare systems. Results continue to support the position that certain source cases readily serve as epicentres of infection.

Fortunately, a rapid global response helped contain the epidemic. However, a high-level international scientific and medical collaboration with intense, rapid sharing of information was needed. This would probably have been impossible without advanced information technology. Quickly, hospital personnel adapted their behavior and treatment schemes. Also, the epidemic was comparatively mild and well contained in countries such as the United States. This is probably due to three factors:

- advance notice of the pandemic;

- an overall quality healthcare system;

- and a heightened nationwide state of preparedness that followed the anthrax-tainted mail scare.

In many ways, the SARS epidemic was handled successfully. Lessons learned during preparedness planning for possible influenza pandemics and for possible bioterrorism attacks proved beneficial. However, shielding of cases in China initially delayed an effective worldwide response.

The SARS experience also demonstrates again the ability of poorly understood infectious disease processes to cause marked worldwide public panic. Anxiety about SARS proved to be larger and moved more quickly than the disease itself. The result could be discrimination against certain people or locations or the improper allocation of limited healthcare resources.

For example, SARS was front-page news for many weeks in the United States, yet few cases were actually noted and no one died. Ironically, the premature deaths of more than 30,000 Americans during the coming influenza season commands little notoriety and even less publicity. 
Table I. Valuable SARS Information Websites

World Health Organization, WHO

www.who.int/csr/sars/en

The Centers for Disease Control and Prevention, CDC

www.cdc.gov/ncidod/sars/

Organization for Safety and Asepsis Procedures, OSAP

www.osap.org/issues/pages/sars/índex.htm?PHPSESSID=6le9lalb380f7ca2d20f9d6a2f69ce5a

American Dental Association, ADA

www.ada.org/prof/pubs/daily/0304/0409sars.html

United States Armed Forces Institute of Pathology

www.afip.org/Departments/Pulmonary/SARS/index.html

United Kingdom Department of Health

www.doh.gov.uk/sars/

Health Canada SARS Site

www.hc-sc.gc.ca/pphb-dgspsp/sars-sras/index.html

Association for Professionals in Infection Control and Epidemiology, APIC

www.apic.org

The Society of Healthcare Epidemiology of America, Inc.

www.shea-online.org/ 
Table 2. Interim US Case Definition of SARS

\section{Clinical Criteria}

- Asymptomatic or mild respiratory illness.

- Moderate respiratory illness:

$\circ$ Temperature of $>38^{\circ} \mathrm{C}\left(100.4^{\circ} \mathrm{F}\right)$ AND

O One or more clinical findings of respiratory illness (e.g. cough, shortness of breath, difficulty breathing or hypoxia)

- Severe respiratory illness:

$\circ$ Temperature of $>38^{\circ} \mathrm{C}\left(100.4^{\circ} \mathrm{F}\right)$ AND

$\bigcirc$ One or more clinical findings of respiratory illness (e.g. cough, shortness of breath, difficulty breathing or hypoxia) AND

- Radiographic evidence of pneumonia OR

- Respiratory distress syndrome OR

- Autopsy findings consistent with pneumonia or respiratory distress syndrome without an identifiable cause.

\section{Epidemiological Criteria}

- Travel (including transit in an airport) within ten days of onset of symptoms to an area with current or previously documented or suspected community transmission of SARS OR

- Close contact within ten days of onset of symptoms with a person known or suspected to have SARS.

\section{Laboratory Criteria}

- Confirmed:

- Detection of antibody to SARS Coronavirus in specimens obtained during acute illness or $\mathbf{2 1}$ days after illness onset OR

- Detection of SARS Coronavirus by reverse transcriptase PCR confirmed by a second PCR assay, by using a second aliquot of the specimen and a different set of PCR primers OR

O Isolation of SARS Coronavirus. 